\title{
VOLUME EFFECT IN THE SILVER VOLTAMETER
}

\author{
By E. B. Rosa and G. W. Vinal
}

In several of our earlier papers ${ }^{1}$ we have described a phenomenon which we have called the "volume effect." This consisted in an excess weight of deposit in the large-size voltameters over that in the smaller sizes which were used simultaneously in series. This difference was seldom very large and was generally attributed to experimental error prior to the middle of February, I9Io. By this time, however, so many cases of the excess deposit in the larger sizes of voltameter (both porous cup and siphon forms) had occurred that a careful analysis of the preceding results was made. The ratio of the deposit in the large-size voltameter to the deposit in the small size was computed for every reliable experiment where large and small voltameters were used in series and were identical in all particulars except the size of the cups and the volume of the electrolyte. Shortly after this we prepared a statement of our results for the International Technical Committee, and briefly discussed ${ }^{2}$ this volume effect. Although we have referred to it more in detail in our later publications ${ }^{3}$ and have given quantitative evidence of its reality and have prepared a theory to account for it, misunderstanding has arisen concerning it and some writers have even questioned its reality. Von Steinwehr ${ }^{4}$ has stated that since we have observed it only in the porous-cup form of voltameter (which, however, is not the case) it must be due to an error caused by the porous cup, and hence the porous cup should be discarded. Richards and Anderegg, ${ }^{5}$ finding their own voltameters (in two experiments) to show an excess deposit in the large size before heating the cups and deposits to redness, concluded that the excess weight was due to mother liquor imprisoned between the surface of the cup and the crystals, the amount being greater in the large cup

1 This Bulletin, 9, pp. 154, 168, 514 .

2 Supplement to Report of the International Committee, p. 9; IgI2.

8 This Bulletin, 10, p. 530; 13, p. I67.

${ }^{4}$ Zs. f. Instrk., 33, p. 32I; 1913.

5 J. Am. Chem. Soc., 37, p. 20; 1915. 
because of the greater surface. More recently, Jaeger and von Steinwehr ${ }^{6}$ have stated that there are no systematic experiments showing the volume effect in any other form of voltmeter but the porous-cup form. We have previously called attention to the fact ${ }^{7}$ that the volume effect was evident in Lord Rayleigh's experiments using the filter-paper voltameter. Averaging all of his comparisons of large and small voltameters there is an outstanding difference of 14.5 in 100000 , the large voltameters usually having the heavier deposit. In only I case out of ro did his small voltameter show an excess of deposit over the large size. Jaeger and von Steinwehr ${ }^{8}$ express the opinion that the accuracy of Lord Rayleigh's results is not sufficient to establish the reality of this difference. This might be granted if there was no other evidence, but at least it tends to confirm the large amount of evidence we shall present below as to the reality of the volume effect.

In dealing with such a phenomenon as the volume effect where the differences are not far removed from the experimental error, we can best treat a large number of observations by statistical methods. Since the volume effect involves a comparison of two voltameter deposits, each of which is subject to an experimental error, it is natural that the experimental error of the ratio should be larger than for the single determinations.

If $S_{1}$ represents the weight of deposit in a large voltameter and $S_{2}$ the weight of deposit in a small voltameter then we may put

$$
Z=\frac{S_{1}}{S_{2}}
$$

If the probable error of $S_{1}$ is $r_{1}$ and of $S_{2}$ is $r_{2}$, then the probable error of $Z$ is $R$ as defined by the equation.

$$
R^{2}=\left(\frac{\delta Z}{\delta S_{1}}\right)^{2} r_{1}^{2}+\left(\frac{\delta Z}{\delta S_{2}}\right)^{2} r_{2}^{2}
$$

Equation (I) after differentiating becomes

$$
R^{2}=\left(\frac{I}{S_{2}}\right)^{2} r_{1}^{2}+\left(\frac{S_{1}}{S_{2}^{2}}\right)^{2} r_{2}^{2}
$$

dividing (2) by $Z^{2}$ we have

$$
\left(\frac{R}{Z}\right)^{2}=\left(\frac{r_{1}}{S_{1}}\right)^{2}+\left(\frac{r_{2}}{S_{2}}\right)^{2}
$$


Each term of (3) represents a percentage probable error. The magnitudes of $\frac{r_{1}}{S_{1}}$ and $\frac{r_{2}}{S_{2}}$ are found by referring to our previous papers. For example, if we use the second series of quantitative results, ${ }^{9}$ we find by a computation 0.0034 per cent for the large size and 0.0026 per cent for the small size. These are slightly higher than for our last and best work. Substituting these values in (3) we obtain as the value of $R$, since $Z$ is practically unity

$$
R=0.0043 \text { per cent. }
$$

This, then, is the probable error of a single determination of the volume effect for the cases in which the salt was used as purchased.

To make the best use of all the data at hand we have subdivided our results into three classes. The first class includes all of our determinations made with the ordinary market variety of c. p. silver nitrate and it includes salt purchased from J. T. Baker \& Co., of America; Baird \& Tatlock, of England; the Gold- und SilberScheide-Anstalt, of Germany; and Poulenc Frères, of France. The second class includes all of our determinations given in our latest and best work of the Part IV ${ }^{10}$ (except where a direct comparison of size of voltameter was not available). In all the observations for class 2 the salt used for preparing the electrolyte was tested by our permanganate and acidity tests ${ }^{11}$ and found satisfactory. Such samples of salt for class I as were tested by these same tests were not found satisfactory. The third class of results includes cases in which the electrolyte was purposely contaminated with filter paper or the extract of filter paper. Seven out of the nine of these determinations were made recently.

The analysis of our observations is perhaps most conveniently and convincingly presented by means of two diagrams. In the first (Fig. I) we have only the results using the porous-cup voltameter, in order that the results so obtained may be compared with those of the second diagram (Fig. 2), which includes the results using the Kohlrausch voltameter (points marked $K$ ), the siphon, the new form of Mr. Smith, and the filter paper form $(F)$. For each diagram the abscissae are ratios of the volume of electrolyte in the large voltameter to that in the small size and the ordinates are ratios of the silver deposit in the large voltameter to that in the small size. Electrolytes used as the salt was purchased without further purification and without satisfying the permanganate and 
acidity tests are designated by solid black dots. The second class-that is, electrolytes made from purified salt which was satisfactory by our tests-is designated by the circles, and the third class, of especially contaminated electrolytes, is represented by

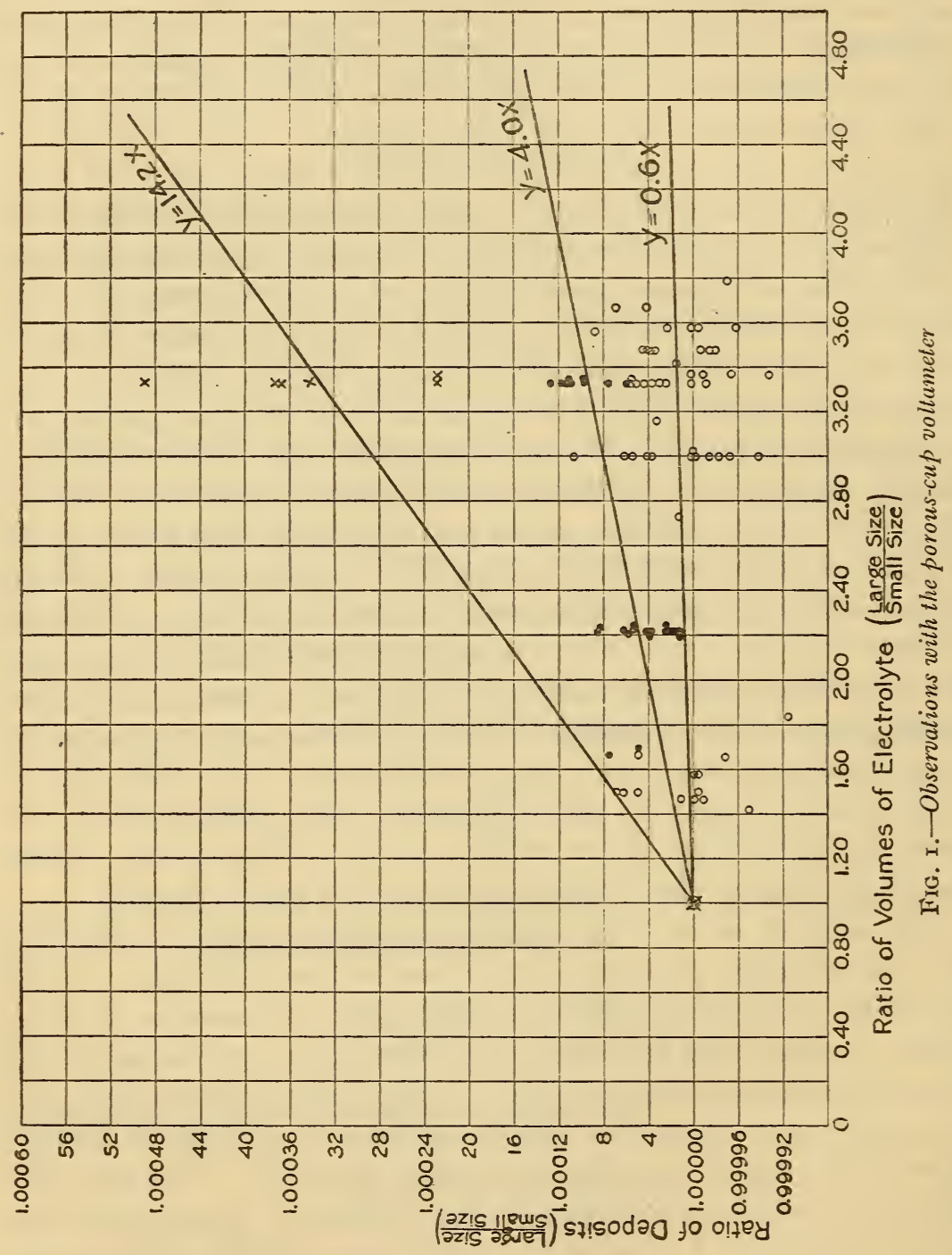

crosses. Each point, therefore, represents the ratio of the deposit in the large size to that in the small size, the two voltameters being exactly similar in all respects but the volume of electrolyte. The only exception to this is in the case of the siphon voltameter. It 
is not practicable to use a siphon voltameter having as small a volume as our ordinary small voltameters ( 90 to $\mathrm{roO} \mathrm{cc}$ ) since the resistance of such an instrument would be too high. In this case, therefore, we have had to compute the ratio of the deposit in the

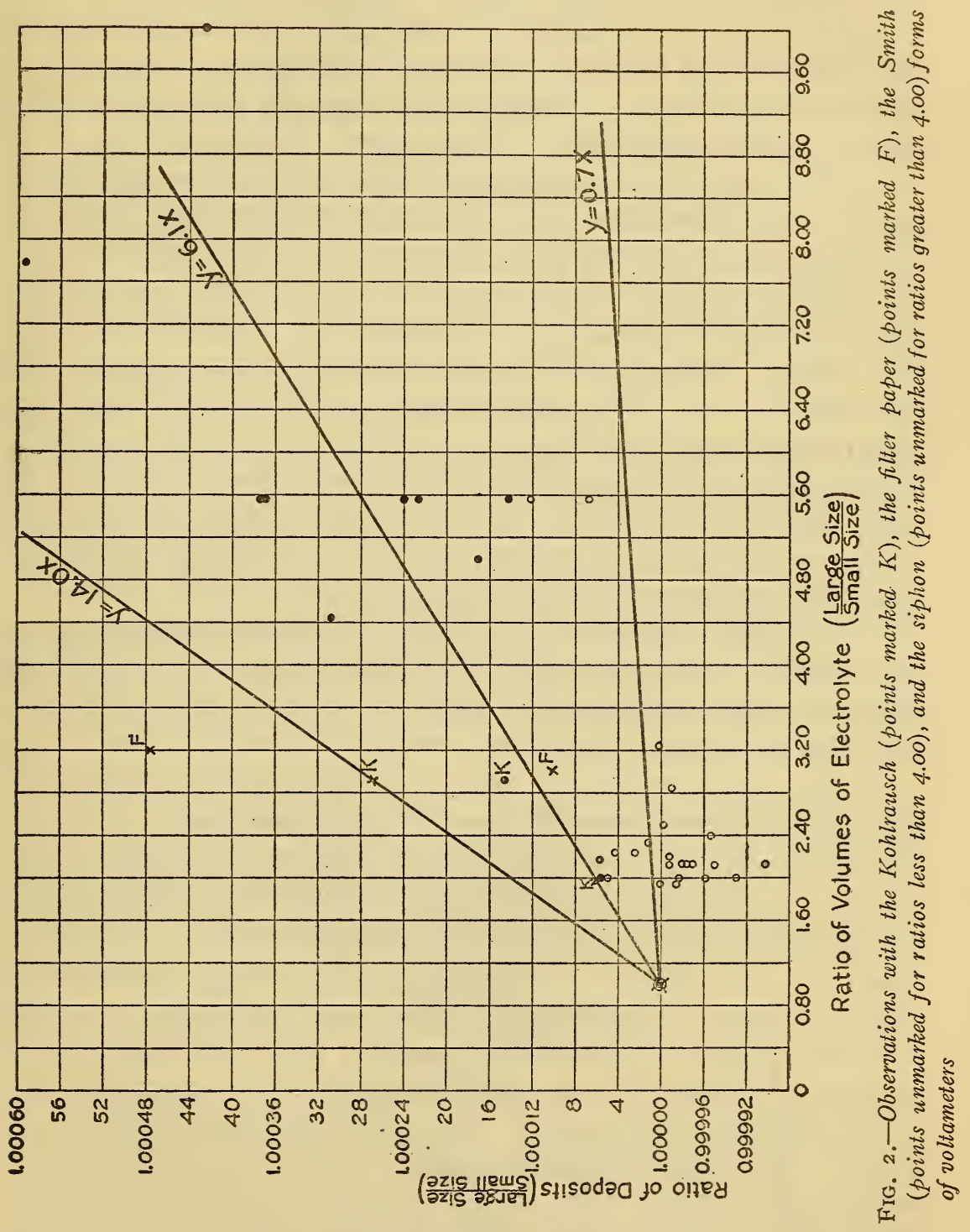

siphon voltameter to the deposit in a small porous-cup voltameter used simultaneously. However, if all the siphon determinations were thrown out on this account the essential facts would remain the same. 
We may now set down certain observations regarding these two diagrams which we think are justified by the results.

I. In no case did the ratio of deposits from electrolyies as purchased fall below unity. This means that the deposit in the largesize voltameter was always greater than in the small size. This is equally true for all the forms of voltameter used. There trere 26 such observations with the porous-cup voltameter, 9 observations with the siphon form, 2 observations with the Kohlrausch form, and I with the new form. Total number of observations, 38 .

2. In the case of pure electrolytes - that is, those represented by circles-the observations show that the deposits in the small voltameters often exceeded those in the large voltameters. In the case of the porous-cup voltameters there are 3 I observations with a ratio of unity or greater and 25 observations with a ratio of less than unity. With the new form, 6 observations are greater than unity and it less. Two observations with the siphon form are greater than unity, and there are no observations at all with the Kohlrausch form. In all there are 39 observations greater than unity and 39 observations less than unity, showing no tendency whatever for the deposit to be heavier in the larger voltameter when the electrolyte is of the highest purity.

3. The observations with electrolytes purposely contaminated all lie far above the value unity. Six observations were made with the porous-cup voltameter, 2 with the filter-paper form, and I with the Kohlrausch form.

4. The above conclusions show that the general facts are the same for all of the different forms of voltameters with the several classes of electrolyte. This is clearly seen by referring to the curves. As the points are necessarily scattered we must depend on the statistical method of treating the observations. Thus, if we compute the equation of the most probable straight line representing porous-cup observations with pure electrolyte we obtain $y=0.6 x$. If we do the same for the new form and siphon observations, we obtain $y=0.7 x$, which is almost perfect agreement and closer than would be expected. The line in each case is nearly horizontal. The slight inclination appears to show that not all the electrolyte was perfectly pure, which of course was hardly to be expected. For the electrolyte as purchased-that is, slightly impure-we compute for the porous-cup voltameter $y=4.0 x$ and similarly for the Kohlrausch, new form, and siphon forms $y=6$.I $x$. These are both very different from the line representing pure electrolytes, the porous-cup form showing, however, a smaller effect. 
Taking the third class of electrolytes-that is, those which have been intentionally contaminated by the addition of filter paper-we compute for the porous-cup observations $y=\mathrm{I} 4.2 x$ and for the Kohlrausch and filter paper forms $y=14.0 x$. This close agreement is of course accidental, but that the line has a much greater slope than for the second class is not accidental.

For convenience these equations have been computed on the assumption that the origin of coordinates is at the point $y=I$, $x=\mathrm{I}$, and they are so given here to avoid the unnecessary complication of a term giving the intercept on the $y$ axis. The value of these equations is merely to show the similarity of the values for the slopes, but if it is desired to write the equations referred to the true origin they can of course be very simply transformed.

If it be objected that because the siphon-voltameter deposits have been computed as the ratio to the deposit in the small porous-cup voltameter, as explained on page 45I, an inspection of figure 2 will show that even omitting all these observations with the siphon no material change would be made in the slope of any of the curves, particularly in the case of the curve $y=6$.I $x$, which includes most of the siphon observations.

5. From the above it appears that our previous statements as to the volume effect were justified. The volume effect is caused by impurities in the electrolyte. It is not confined to the porouscup voltameter, but occurs with all types, and is greater when the impurities in the electrolyte are greater and disappears when the electrolyte is strictly pure.

6. In a previous paper Vinal and Bovard ${ }^{12}$ showed that the volume effect was not due to greater inclusions in the large-size voltameter, as supposed by Richards and Anderegg. ${ }^{13}$

We give in the table below some results recently obtained at the Bureau.

TABLE 1

\begin{tabular}{|c|c|c|c|c|c|c|}
\hline \multirow{2}{*}{ Form } & \multicolumn{3}{|c|}{ Contaminated } & \multicolumn{3}{|c|}{ Uncontamirated } \\
\hline & Large & Small & $\Delta$ & Large & Small & 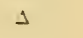 \\
\hline & $\mathrm{mg}$ & $m g$ & $\operatorname{mg}$ & $\mathrm{mg}$ & . mg & mg \\
\hline Filter paper. & 4212.00 & 4210.00 & 2.00 & 4206.02 & 4205.07 & 0.95 \\
\hline Do......... & 4069.59 & 4069.18 & .41 & 4067.75 & 4068.19 & -.44 \\
\hline 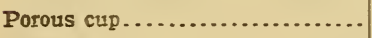 & 4492.27 & 4491.06 & 1.21 & 4489.83 & 4489.49 & .34 \\
\hline Kohlrausch..... & 4244.26 & 4243.12 & 1.14 & 4241.99 & 4241.37 & .62 \\
\hline Porous cup. & 4277.83 & 4276.86 & .97 & 4275.99 & 4275.52 & .47 \\
\hline Porous cap. & & $a 4276.72$ & 1.11 & & & \\
\hline
\end{tabular}


The electrolyte used was an ordinary sample of c. p. silver nitrate, without further purification. This is marked "uncontaminated." It is seen from the above that this salt produced a volume effect of about $0.40 \mathrm{mg}$ on the average, although the results with the filter-paper form are erratic, as might be expected. A portion of the same salt contaminated with filter paper was electrolyzed in two similar voltameters, and the results are given in the column headed "contaminated." It is seen that the effect is increased three times, on the average. In the last experiment we used the smallest porous-cup voltameter that we could arrange for a $4 \mathrm{~g}$ deposit. The electrolyte was only $40 \mathrm{cc}$. The table shows that it gave a smaller deposit than in what we have ordinarily called the small size ( $90-100 \mathrm{cc}$ ). The large-size voltameters contained about $300 \mathrm{cc}$. These volumes, in the case of the porous-cup voltameters, mean the volume of electrolyte outside the porous cup. We showed in a previous place ${ }^{14}$ how effective a barrier the porous cup is between the anode and cathode.

It will be noticed in the table that the deposits from the contaminated solutions, although differing between themselves according to size, are all considerably inexcess of even the largesize voltameter containing the uncontaminated electrolyte. The reason for this will appear after a brief account of what we regard as the mechanism of this volume effect.

If we add some of the filter-paper extract ${ }^{15}$ to a solution of silver nitrate in water, or put the filter paper directly in the electrolyte, marked changes in the condition of the solution take place. The filter-paper extract is a basic organic colloid ${ }^{16}$ which produces a reducing action ${ }^{17}$ on the silver nitrate. As the reduction proceeds important changes in the acidity ${ }^{18}$ of the solution take place and the reduced silver appears in colloidal form. ${ }^{19}$ It may become visible as a colloidal solution in cases where the filter paper contamination is considerable. The colloidal particles travel slowly to the cathode ${ }^{20}$ under the influence of the electric current, and they are also helped along in this direction by the convection currents of liquid. The denser liquid from the region of the anode falls to the bottom of the cup and displaces upward the impoverished liquid at the cathode surface. ${ }^{21}$ Probably each particle of colloidal silver drags along with it some of the organic colloid,

15 This Bulletin, 9, pp. 20r, 220, 510.
15 This Bulletin, 9, p. 237.
is This Bulletin, 9, p. 239 .
${ }^{37}$ This Bulletin, 9, p. 23\%.
18 This Bulletin, 9, p. 247

19 This Bulletin, 9, p. 238 .

20 This Bulletin, 9, p. 2 I9

21 This Bulletin, 9. p. 269. (See Fig. 27 of Part II.) 
which acts as a protective colloid ${ }^{22}$ and prevents the rapid precipitation of the silver by the unreduced electrolyte.

In the contaminated electrolyte we have, therefore, a very large number of silver particles each of which is an aggregate of a large number of silver atoms. These are gradually swept along toward the cathode, and as each particle reaches the cathode it deposits its load of silver and organic matter ${ }^{23}$ and also delivers up its charge, but the amount of electricity so transported is not commensurate with the mass of material laid down on the cathode. In this way an excess weight of deposit over that calculated for a univalent ion is produced, and this may be further increased by a somewhat greater inclusion ${ }^{24}$ of the mother liquor, which is made possible by the colloid breaking up the crystalline structure of the deposits.

The rate at which this colloidal matter is swept out of the solution will depend upon the density of the electric current. At the beginning of an experiment we may justly assume that the number of colloidal particles per cubic centimeter of the electrolytes in contact with the cathode is the same in both large and small voltameters. When the electric current starts, the greater intensity of the current in the small voltameter due to the smaller cathode area will sweep the colloidal particles out of the solution at a greater rate than in the large size. Consequently, the electrolyte contiguous to the cathode in the small voltameter will become impoverished as to colloidal matter; that is, it is purified. The number of colloidal particles which will subsequently deposit per second diminishes for lack of the colloidal matter. However, the effect of the electric current and the convection currents of liquid, which arise because of the changing densities of the solution at the anode and cathode will replenish, impart, the colloidal matter to the depleted cathode solution. In the large voltameter the forces drawing the colloidal matter out of the solution at the cathode are less severe, as we have mentioned above, and in addition the convection currents of liquid are less impeded by the anode and its porous cup or glass trap, so that the solution on the face of the cathode is freed from colloidal matter more slowly than in the small voltameter. There is, of course, a greater supply of the colloidal particles in the voltameter having the greater volume of electrolyte. It is reasonable, therefore, to anticipate that more colloidal particles will be deposited in a large voltameter than in a small one during the two hours that the current flows. Conse- 
quently, the deposit in the large cup will be heavier than the deposit in the small cup. (See Fig. 3.)

Probably there are some colloidal particles even in our purest solutions. We have never found a solution, on examination by the ultramicroscope, entirely devoid of colloidal particles; but in a strongly contaminated solution the colloidal particles are increased in number thousands of times. It is not surprising, there-

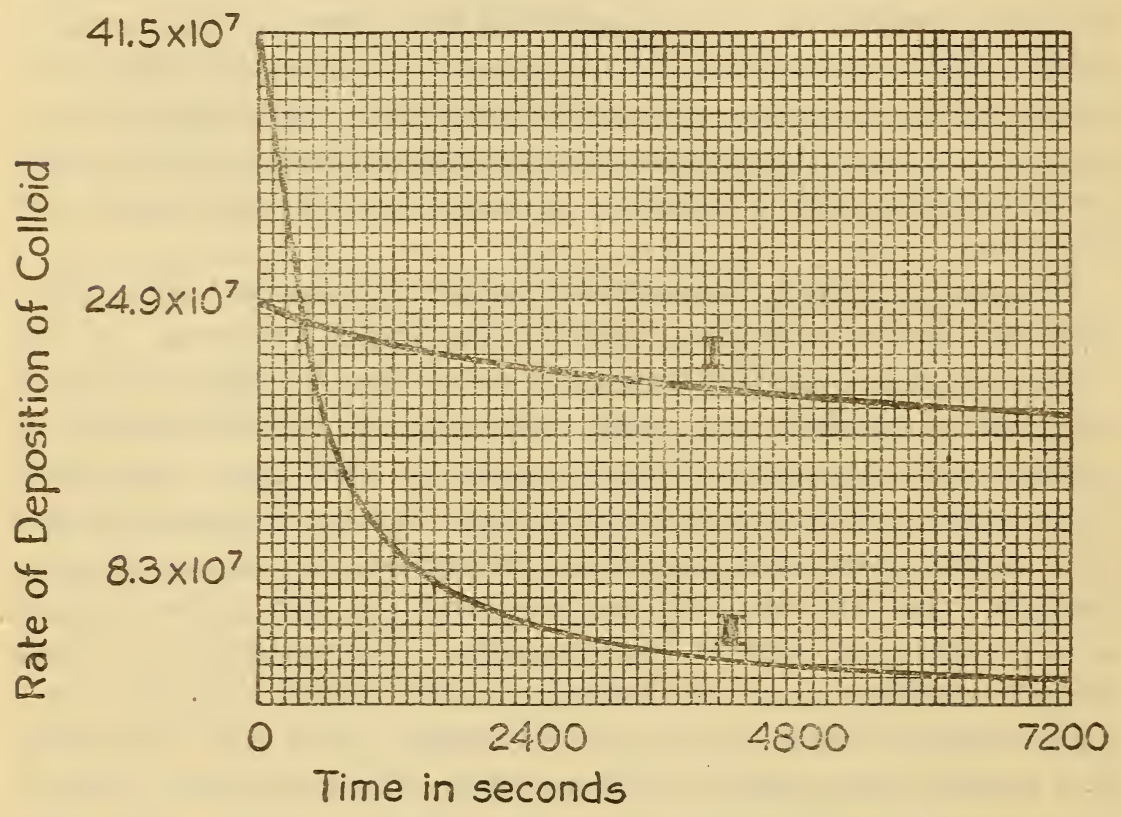

FIG. 3.- Hypothetical curves illustrating the theory of the volume effect

These curves are based on an average case in which the excess of deposit in the large voltameter over that in the small voltameter is $0.65 \mathrm{mg}$. It is assumed that the average diameter of the colloidal particles is $0.05 \mu$ from which we compute that $\mathrm{I} \times$ Io $^{12}$ more particles are deposited on the large cathode than on the small cathode. The area under each curve represents the supposed number of particles deposited on each cathode and the curves have been dawn so that there are rooo more square divisions under curve I, for the large voltameter, than under curve II, for the small voltameter. Each division, therefore, represents $10^{9}$ particles. The rate at which the particles deposit is initially greater in the small voltameter, because the electric intensity at the cathode is greater, due to the smaller area, but as the available supply of particles is more limited than it the larger size, the curve falls off more rapidly, and at the end of the experiment the total number of particles deposited in the large voltameter is greater than in the small one.

fore, that even in the small voltameter we should find an excess of deposit over that in voltameters which contain pure or nearly pure solution. The last experiment given in the table shows that the very small voltameter gave a smaller deposit than our ordinary small size with impure electrolyte; but even this is in excess of the large voltameter containing purer electrolyte, which, however, was not quite pure. 
In our earlier work ${ }^{25}$ we showed that a contaminated solution, giving initially a strongly striated deposit, became purer as the electrolysis proceeded, until in the end the deposit was a normal crystalline deposit such as we always find from a pure electrolyte.

We think that the foregoing explanation of the volume effect is in accord with all the experimental facts and that it is a reasonable and logical explanation. It shows that using the same cathodes and anodes and porous cups the volume effect may be made to appear or disappear according as the electrolyte is contaminated or not, and that using the same cathode and solution the volume effect may be increased by increasing the volume of electrolytes (that is, increasing the amount of colloid present) as is done when the siphon voltameters are used. The theory of Richards and Anderegg ${ }^{26}$ assuming the effect to be a matter of greater inclusions on a greater cathode area, will explain neither of the above facts. But these facts have been demonstrated over and over again in our work. Our theory of the volume effect also shows that it is not a phenomenon of the porous-cup voltameter alone, as stated by von Steinwehr, ${ }^{27}$ but is common to all forms. The diagrams which we have given above show that this is the case.

One further experiment was, however, tried to answer the question of whether the relatively dilute solution at the cathode surface could produce a false weight of deposit by unloading silver chloride, since it is well known that silver chloride is more soluble in strong silver-nitrate solutions than in weak ones. This has been clearly shown in a quantitative way recently by Lowry. ${ }^{28}$ Accordingly, we prepared a ro per cent solution of silver nitrate and saturated one portion of it with silver chloride, allowing the finely suspended particles to settle out during 48 hours, when the clear, supernatant liquid was decanted without filtration and electrolyzed in a large and small voltameter simultaneously with the portion not containing the silver chloride. No-differences due to the chloride were found.

We believe that a careful study of the above data, giving the results of a large number of experiments, will lead to the conclusion that the reality and significance of the volume effect have been proved beyond question, and that it is a valuable criterion of the purity of the electrolyte.

WASHINGTON, March 28, igr6. 